\title{
Comprehensive analysis of formin gene family highlights candidate genes related to pollen cytoskeleton and male fertility in wheat (Triticum aestivum L.)
}

Wen-jing Duan ${ }^{1,2,3 \dagger}$, Zi-han Liu' ${ }^{1,2+}$, Jian-fang Bai ${ }^{1,2+}$, Shao-hua Yuan ${ }^{1,2}$, Yan-mei Li ${ }^{1,2}$, Feng-kun $\mathrm{Lu}^{1,2,3}$, Tian-bao Zhang ${ }^{1,2}$, Jia-hui Sun ${ }^{1,2}$, Feng-ting Zhang ${ }^{1,2^{*}}$, Chang-ping Zhao ${ }^{1,2^{*}}$ and Li-ping Zhang ${ }^{1,2^{*}}$

\begin{abstract}
Background: Formin, a highly conserved multi-domain protein, interacts with microfilaments and microtubules. Although specifically expressed formin genes in anthers are potentially significant in research on male sterility and hybrid wheat breeding, similar reports in wheat, especially in thermo-sensitive genic male sterile (TGMS) wheat, remain elusive.

Results: Herein, we systematically characterized the formin genes in TGMS wheat line BS366 named TaFormins (TaFHs) and predicted their functions in inducing stress response. In total, 25 TaFH genes were uncovered, majorly localized in 2A, 2B, and 2D chromosomes. According to the neighbor-joining ( $\mathrm{NJ}$ ) method, all TaFH proteins from wheat and other plants clustered in 6 sub-groups (A-F). The modeled 3D structures of TaFH1-A/B, TaFH2-A/B, $\mathrm{TaFH} 3-\mathrm{A} / \mathrm{B}$ and TaFH3-B/D were validated. And different numbers of stress and hormone-responsive regulatory elements in their 1500 base pair promoter regions were contained in the TaFH genes copies. TaFHs had specific temporal and spatial expression characteristics, whereby TaFH1, TaFH4, and TaFH5 were expressed highly in the stamen of BS366. Besides, the accumulation of TaFHs was remarkably lower in a low-temperature sterile condition (Nanyang) than fertile condition (Beijing), particularly at the early stamen development stage. The pollen cytoskeleton of BS366 was abnormal in the three stages under sterile and fertile environments. Furthermore, under different stress levels, TaFHs expression could be induced by drought, salt, abscisic acid (ABA), salicylic acid (SA), methyl jasmonate (MeJA), indole-3-acetic acid (IAA), polyethylene glycol (PEG), and low temperature. Some miRNAs, including miR167, miR1120, and miR172, interacts with TaFH genes; thus, we constructed an interaction network between microRNAs, TaFHs, phytohormone responses, and distribution of cytoskeleton to reveal the regulatory association between upstream genes of TaFH family members and sterile.
\end{abstract}

\footnotetext{
*Correspondence: lyezh@163.com; cp_zhao@vip.sohu.com;

Ipzhang8@126.com

tWen-jing Duan, Zi-han Liu and Jian-fang Bai contributed equally to this work.

'Beijing Engineering and Technique Research Center for Hybrid Wheat, Beijing Academy of Agriculture and Forestry Sciences, Beijing 100097, China Full list of author information is available at the end of the article
}

(c) The Author(s). 2021 Open Access This article is licensed under a Creative Commons Attribution 4.0 International License, which permits use, sharing, adaptation, distribution and reproduction in any medium or format, as long as you give appropriate credit to the original author(s) and the source, provide a link to the Creative Commons licence, and indicate if changes were made. The images or other third party material in this article are included in the article's Creative Commons licence, unless indicated otherwise in a credit line to the material. If material is not included in the article's Creative Commons licence and your intended use is not permitted by statutory regulation or exceeds the permitted use, you will need to obtain permission directly from the copyright holder. To view a copy of this licence, visit http://creativecommons.org/licenses/by/4.0/ The Creative Commons Public Domain Dedication waiver (http://creativecommons.org/publicdomain/zero/1.0/) applies to the data made available in this article, unless otherwise stated in a credit line to the data. 
Conclusions: Collectively, this comprehensive analysis provides novel insights into TaFHs and miRNA resources for wheat breeding. These findings are, therefore, valuable in understanding the mechanism of TGMS fertility conversion in wheat.

Keywords: Wheat, Thermo-sensitive genic male sterile, Formin gene family, Cytoskeleton, MiRNA

\section{Background}

Eukaryotic cells possess a dynamic actin cytoskeleton that controls cell growth, division, and polarity formation by alternating its global and filamentous state in response to developmental and environmental stimuli [1]. Formins (formin homology proteins), a group of proteins involved in actin polymerization, are associated with the fast-growing end (bared end) of actin filaments, potentially mediating a series of cellular functions, including polarity, division, cytokinesis, migration, among others. Several formins have been identified in plants. The formin proteins are characterized by two formin homology (FH) domains, FH1 and FH2. The FH1 (formin homology 1) domain harbors a distinct polyproline-rich region that binds to a crucial actin-binding proteinprofilin or actin/profilin complex. Profilin is a vital monomer actin-binding protein and can interact with formin via the FH1 domain. The FH2 (formin homology 2) domain is characterized by a key sequence that interacts with actin [2]. Plant formins occur in two categories, type I and type II, based on the sequence homology of their FH (formin homology) domains. Type I formins have a transmembrane (TM) domain at their $\mathrm{N}$-terminus followed by the C-terminal $\mathrm{FH} 1$ and $\mathrm{FH} 2$ domains, whereas type II formins do not have an N-terminal TM domain but carry an N-terminal phosphatase and tensinrelated (PTEN)-like domain besides the conserved FH1 and $\mathrm{FH} 2$ domains [3].

Also, formins have multiple members in each species; for example, 22 formin family members are present in the Arabidopsis genome. Specifically, AtFH1, AtFH5, AtFH6, and AtFH8 have been described in Arabidopsis and their functions explored in vivo. For instance, AtFH1 overexpression in pollen tubes induced the formation of arrays of actin cables, projecting into the cytoplasm from the cell membrane. Interestingly, AtFH1 expression induced tube broadening, growth depolarization, and growth arrest in transformed pollen tubes. With these observations, it was suggested that AtFH1 regulation of actin polymerization could be necessary for the polarized pollen cell growth process [4]. The AtFH5-GFP fusion protein accumulated in the cell plate, essential for cell division. Furthermore, AtFH6 regulated polarized growth by tuning the assembly of actin cables [5]. Other reports revealed that AtFH8 affects root and root hair development by alternating actin cytoskeleton distribution $[6,7]$. Collectively, formins function as key factors potentially bridging several vital pathways related to plant pollen growth and development, particularly cytoskeleton distribution. Notably, formins have been adequately characterized in animals, yeast, and Arabidopsis. For example, the first gene encoding forming - the mouse limb deformity (ld) gene in 1982, the mutation of which led the mice to fail to "form" proper limbs and kidneys, and a large family of formin homology proteins were related with the field of actin cytoskeleton $[8,9]$. However, the formin gene family members are yet to be uncovered in the wheat genome. Also, it is not clear whether formin genes are related to pollen development, especially in the distribution of microspore cytoskeleton in wheat.

Wheat is one of the most important staple food crops in China and globally in terms of production and consumption [10]. Given that wheat production varies with climate and other environmental conditions, application of molecular biology and genetics methods is critical for advancing the stress tolerance and quality of wheat. Thermo-sensitive genic male sterile wheat (TGMS) lines, such as BS366, are of particular significance in a twoline hybrid system. The lines are highly efficient in breeding [11] because their fertility is strictly temperature-dependent [12-14]. Hybrid seeds can be produced under sterile conditions (TGMS line as the maternal plant), whereas the TGMS line can be reproduced under fertile conditions [15]. Thus, the BS366 is an ideal material for studying the genetic mechanism of TGMS and realize the effective adoption of hybrid wheat. In our previous cytological findings, the male sterility in the TGMS wheat cultivar BS366 was primarily induced by the disordered cytoskeleton distribution during meiosis of microsporocytes [16]. However, no study has reported on the contribution of formin gene family members related to pollen cytoskeleton distribution in TGMS wheat. Additionally, miRNAs are one type of small RNAs, which directly mediate the expression of target genes through post-transcriptional negative regulation [17]. Further information on miRNA association with the formin gene family is scanty. Therefore, there is a need to comprehensively explore the formin gene family in wheat and elucidate the interaction between TaFHs and miRNA; this would reveal their potential roles during anther development inBS366.

In this study, after identifying the formin genes of wheat $(T a F H s)$, we evaluated their characteristics for the physicochemical, phylogenetic relationship, 3D structure, 
motif structure, exon/intro structure, chromosomal location, miRNA regulation, and expression patterns in different tissues and different development stages of anthers in the TGMS wheat line, BS366. The results presented will enrich our knowledge of the TaFH genes family and offer the theoretical basis and novel candidate genes for improving and developing the male sterility wheat lines. It is imperative for us to understand the fertility pathway of TGMS.

\section{Results}

\section{Identifying formin genes in wheat}

Two strategies were adopted to mine for formin genes in T. aestivum L. BLASTP using the formin of $A$. thaliana proteins as queries and HMMER searches using the formin domain as a query against the T. aestivum L. protein database. NCBI-CDD and SMART were used to confirm whether the formin domains were present. In total, 25 formin genes were identified, denoted as TaFH1 to TaFH10, based on their location on the chromosomes. Based on the physicochemical analysis of the protein sequence encoded by the wheat formin gene, there were significant differences in physical and chemical properties across the members of the wheat formin family. The protein comprised 306-1403 amino acids in length, with a molecular weight of 24.40-153.70 and 5.3-9.86 isoelectric points. Subcellular localization predictions revealed that $\mathrm{TaFH}$ proteins functioned in cytoplasmic, nuclear, vacuolation, plasma membrane, chloroplast, and endoplasmic reticulum (Table 1). Different characteristics of TaFH genes and proteins were also revealed. Results demonstrated that different $\mathrm{TaFH}$ proteins exerted potentially distinct biological functions.

\section{Phylogenetic tree and domain analysis of TaFHs}

To elucidate the phylogeny of formin proteins, we adopted the MEGA software to analyze the 25 identified formin genes using an evolutionary tree. Based on the information on the phylogenetic relationship, $25 \mathrm{TaFH}$ genes were clustered into 10 groups (Fig. S1). Results demonstrated that $25 \mathrm{TaFH}$ genes could be classified into four groups; the large group could then be

Table 1 Characteristics of the formin gene family members in wheat

\begin{tabular}{|c|c|c|c|c|c|c|c|}
\hline Genes & Sequence ID & Location & length/aa & length/bp & $\mathrm{MW}(\mathrm{KDa})$ & pl & Subcellular Localization \\
\hline TaFH1-A & TraesCS1A02G075600.1 & 1A: $58701490: 58716429$ & 1388 & 4167 & 151.97 & 7.02 & cytoplasm \\
\hline TaFH1-B & TraesCS1B02G094200.1 & 1B: $95651637: 95653727$ & 1403 & 4214 & 153.70 & 7.02 & cytoplasm \\
\hline TaFH1-D & TraesCS1D02G077900.1 & 1D: 59944922:59946998 & 1401 & 4206 & 153.21 & 8.09 & cytoplasm \\
\hline TaFH2-A & TraesCS2A02G388800.1 & 2A: $636904233: 636910538$ & 382 & 1149 & 42.62 & 6.42 & cytoplasm \\
\hline TaFH2-B & TraesCS2B02G406800.1 & 2B: 576081966:576083651 & 932 & 2799 & 104.47 & 6.01 & cytoplasm \\
\hline TaFH2-D & TraesCS2D02G386600.1 & 2D: $491802415: 491808742$ & 384 & 1155 & 42.85 & 6.12 & cytoplasm \\
\hline TaFH3-A & TraesCS2A02G183000.1 & 2A: $142713110: 142717769$ & 408 & 1227 & 45.75 & 8.95 & nuclear \\
\hline TaFH3-B & TraesCS2B02G209600.1 & 2B: 191747275:191752068 & 408 & 1227 & 45.75 & 8.95 & nuclear \\
\hline TaFH3-D & TraesCS2D02G190800.1 & 2D: 134795881:134800421 & 408 & 1227 & 45.75 & 8.95 & nuclear \\
\hline TaFH4-A & TraesCS2A02G214500.1 & 2A: 200622432:200625109 & 946 & 2841 & 101.96 & 7.21 & vacuolation \\
\hline TaFH4-B & TraesCS2B02G239500.1 & 2B: 243786328:243788946 & 941 & 2826 & 101.09 & 6.61 & plasma membrane \\
\hline TaFH4-D & TraesCS2D02G220300.1 & 2D: $187644176: 187646539$ & 957 & 2904 & 103.29 & 6.03 & plasma membrane \\
\hline TaFH5-A & TraesCS2A02G044700.1 & 2A: $17618132: 17619862$ & 1038 & 3117 & 107.62 & 7.22 & chloroplast \\
\hline TaFH5-B & TraesCS2B02G056800.1 & 2B: 27710139:27711846 & 1048 & 3147 & 108.88 & 6.49 & chloroplast \\
\hline TaFH6-A & TraesCS3A02G397900.1 & 3A: $645131154: 645133017$ & 949 & 2850 & 101.05 & 9.42 & plasma membrane \\
\hline TaFH6-B & TraesCS3B02G429900.1 & 3B: 669426515:669428377 & 954 & 2865 & 101.58 & 9.45 & plasma membrane \\
\hline TaFH6-D & TraesCS3D02G391800.1 & 3D: 507028317:507030177 & 952 & 2859 & 96.03 & 9.42 & plasma membrane \\
\hline TaFH7-A & TraesCS4A02G047000.1 & 4A: $38641338: 38643658$ & 890 & 2673 & 95.22 & 9.06 & vacuolation \\
\hline TaFH7-D & TraesCS4D02G257700.1 & 4D: 427461005:427463346 & 886 & 2661 & 94.82 & 9.06 & plasma membrane \\
\hline TaFH8-A & TraesCS5A02G075500.1 & 5A: $90913931: 90925317$ & 306 & 921 & 34.40 & 5.3 & chloroplast \\
\hline TaFH9-B & TraesCS6B02G389300.1 & 6B: 664230386:664232873 & 1336 & 4011 & 145.50 & 6.37 & chloroplast \\
\hline TaFH9-D & TraesCS6D02G339300.1 & 6D: 438905707:438908215 & 1335 & 4008 & 145.46 & 6.5 & endoplasmic reticulum \\
\hline TaFH10-A & TraesCS6A02G312000.1 & 6A: 548402427:548404312 & 829 & 2490 & 86.92 & 9.86 & chloroplast \\
\hline TaFH10-B & TraesCS6B02G342200.1 & 6B: 602822952:602824912 & 901 & 2706 & 94.01 & 9.72 & chloroplast \\
\hline TaFHIO-D & TraesCS6D02G291400.1 & 6D: 402117660:402119621 & 901 & 2706 & 94.22 & 9.72 & chloroplast \\
\hline
\end{tabular}


subdivided into four groups. The conservative domains of these domain were the regions where genes function. The TaFHs structure were obtained and the number of exons and introns were highly divergent (Fig. S2). Most members of TaFHs shared the similar patterns of exon/ intro structure, including intro phase, intro number and exon length.

To reveal the functional information of $\mathrm{TaFH}$ genes, we constructed a phylogenetic tree based on the comparison among the $T$. aestivum, $P$. patens, $S$. moellendorffii, $P$. trichocarpa, S. bicolor, B. distachyon, A. thaliana, and $O$. sativa (Fig. 1). All the formin proteins clustered into six groups, except for the PpFH2, PpFH4 and PpFH5. In total, 25 sequences of 10 members of the wheat formin gene family clustered into six subgroups. Moreover, we found that the $\mathrm{TaFH}$ proteins were clustered into the same clades with some $\mathrm{BdFH}, \mathrm{SbFH}$ and OsFH proteins. For example, TaFH2, BdFH12, SbFH12 and OsFH8 were clustered into group A, BdFH1, SbFH6 and $\mathrm{OsFH} 5$ were clustered with $\mathrm{TaFH} 3$ on the group $\mathrm{B}$, TaFH4 and TaFH5 were clustered into the group D with BdFH2, BdFH11, SbFH5, SbFH11, OsFH4 and OsFH3, and TaFH9 were clustered into the group $\mathrm{E}$ with $\mathrm{BdFH}$, $\mathrm{SbFH} 10$ and OsFH1. These showed that TaFH proteins had a close correlation with those in B. distachyon, $S$. bicolor and $O$. sativa and shared a low similarity with those in P. patens.

In this study, eight $\mathrm{TaFHs}$ representative proteins were modeled using homology to predict the 3D structure. The Phyre 2 server and PyMDLWin been used to deduced and visualized, which were shown in Fig. S3. The results also shown that protein structure of $\mathrm{A}, \mathrm{B}$ and $\mathrm{D}$ homoeologous copies with TaFH1-A/B, TaFH2-A/B, TaFH3-A/B and TaFH9-B/D had shown significant similarity. The 3D structure of $\mathrm{TaFH}$ proteins laid a foundation to explor the complex mechanism of formins at the structure level.

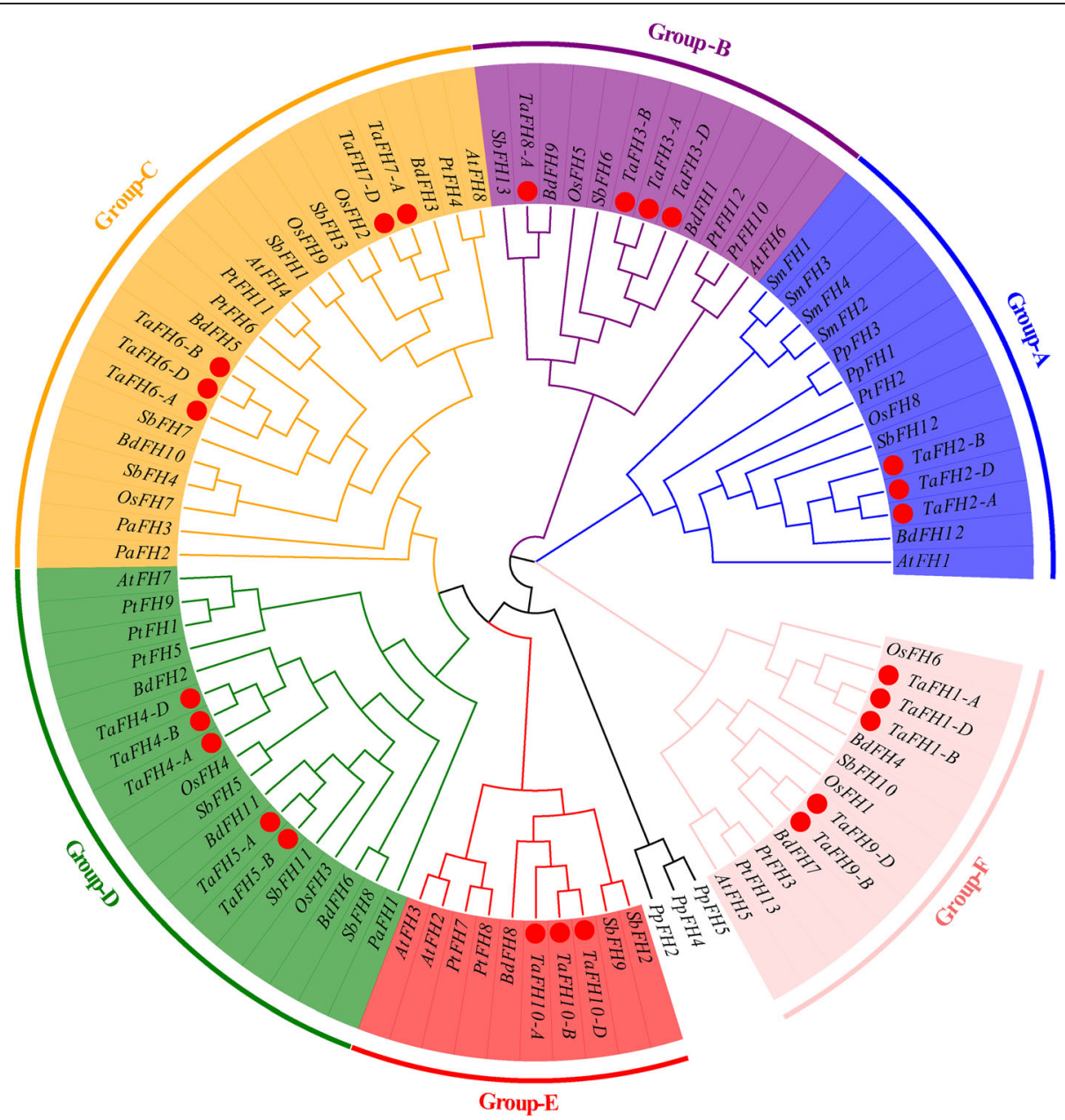

Fig. 1 The phylogenetic tree of TaFH and other species proteins. The tree was constructed from a complete alignment of formins from $T$. aestivum (Ta), P. patens (Pp), S. moellendorffii (Sm), P.trichocarpa (Pt), S. bicolor (Sb), B. distachyon (Bd), A.thaliana (At), and O. sativa (Os), then using MEGA 6.0 by the neighbor-joining method with 1000 bootstrap replicates; bootstrap scores are depicted on the nodes 


\section{Chromosomal assignment of $\mathrm{TaFH}$ genes}

Map files were constructed based on the location of the wheat formin gene family on the chromosome. A chromosome distribution map was drawn using the MapInspect software. A total of 25 wheat formin genes were located on the chromosomes (Fig. 2). Three copies of TaFH1, TaFH2, TaFH3, TaFH4, TaFH6, and TaFH1O were located on sub-genomes $\mathrm{A}, \mathrm{B}$, and D of wheat chromosomes 1, 2, 3, and 6. Two copies of TaFH5, TaFH7, and TaFH9 were located on sub-genomes of wheat chromosomes 2, 4, and 6, respectively. TaFH8 had one copy on the short arm of chromosome 5A. Four formin genes were found on chromosomes $2 \mathrm{~A}$ and $2 \mathrm{~B}$, and three formin genes on chromosome $2 \mathrm{C}$, existing in clusters. However, no formin gene was present on $7 \mathrm{~A}, 4 \mathrm{~B}, 5 \mathrm{~B}, 7 \mathrm{~B}, 5 \mathrm{D}$, and 7D, and this demonstrated a widespread but uneven distribution of the wheat formin genes on the wheat chromosome.

\section{MicroRNA targeting prediction of $\mathrm{TaFH}$ genes}

To further understand the function of TaFH genes, we evaluated the binding association of the TaFH genes with miRNAs. Results revealed that 19 of the $25 \mathrm{TaFH}$ genes harbored targets for 13 different miRNAs (Fig. 3 and Table S1). We also found that tae-miR167a, taemiR167b, and tae-miR167c-5p potentially target TaFH2$A, T a F H 2-B$, and TaFH2-D. Indeed, Tae-miR167 has been shown to target other wheat genes. For example, tae-miR167a directly targeted Auxin Response Factor 8
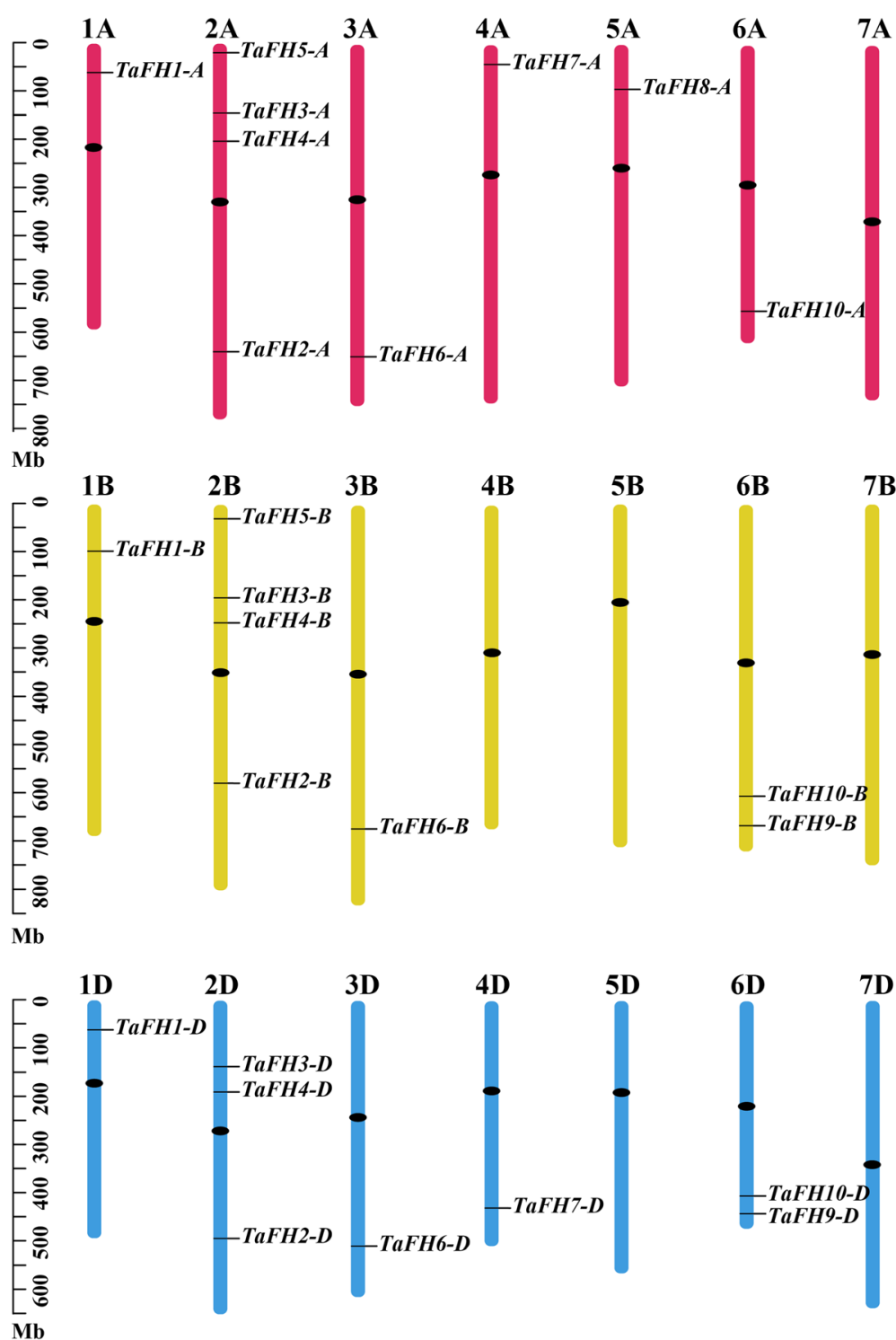

Fig. 2 Chromosomal assignment of the formin gene family in wheat. The chromosome numbers are indicated at the top of each bar, whereas the size of a chromosome is denoted by the unit of the left scale with Mb. Black dots on the chromosome denotes the position of the centromeres 


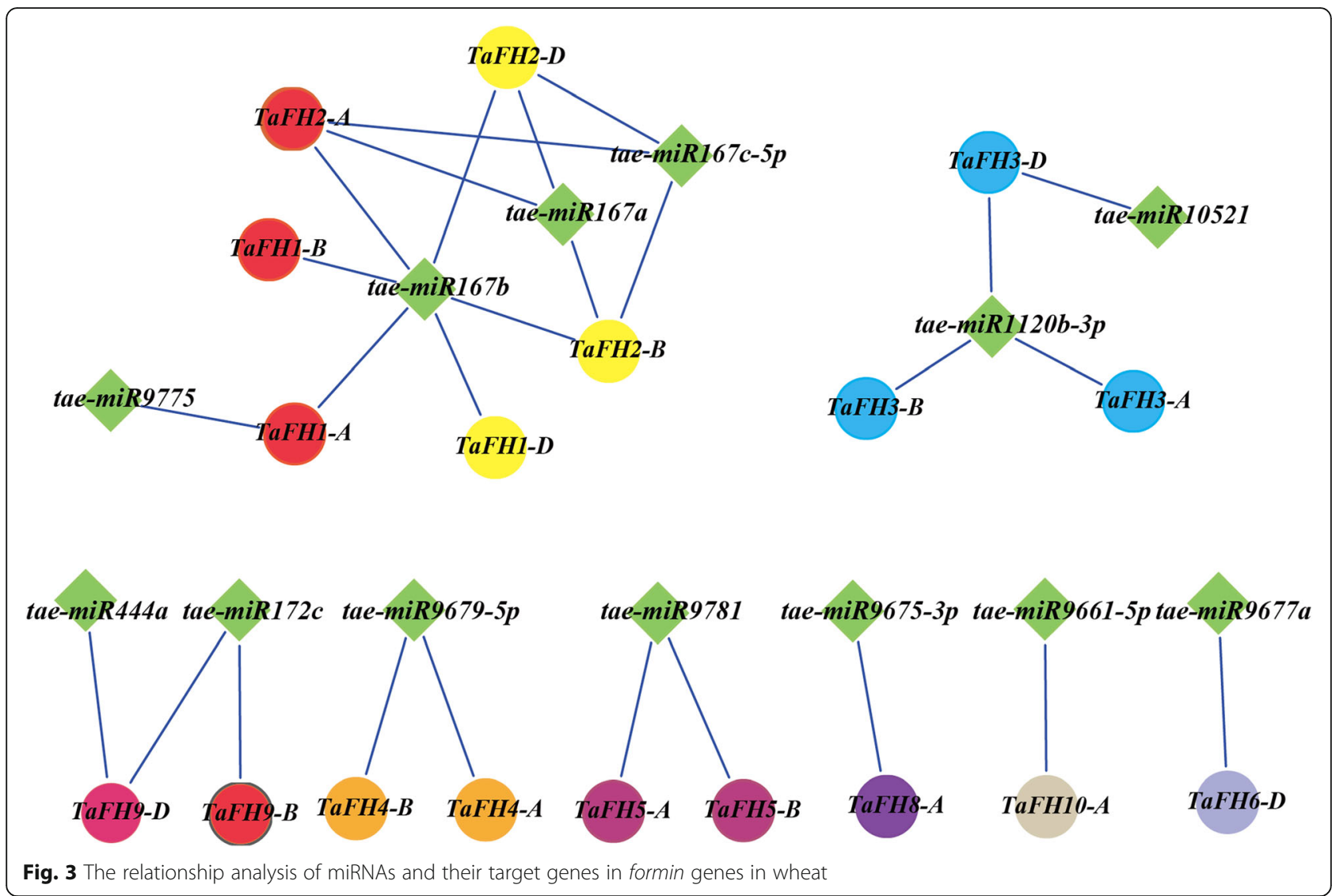

(TaARF8), which possibly regulated auxin biosynthesis by impeding the downstream genes. Also, tae-miR167a overexpression in Arabidopsis lowered the expression levels of its targets (AtARF6 and AtARF8) and induced male sterility phenotypes [18]. Our results suggested that tae-miR167b might target TaFH1-A, TaFH1-B, and TaFH1-D. Tae-miR1120b-3p has been shown to target TaFH3- $A$ and TaFH3-B, and possibly $L R R$, thereby regulating the jasmonate biosynthesis or auxin response pathway associated with pollen development. A previous study found that the $Q$ gene, involved in bread wheat spike architecture, was suppressed by tae-miR172 [19]. In a separate study, Tae-miR444a targeted TaFH9-D and regulated floral patterning and development via the transcription factor MADS-box [20]. Collectively, the findings of this study could help to further understand the regulation between miRNA and formin in the related TGMS pathway.

\section{Promoter analysis of $\mathrm{TaFH}$ genes}

To establish how the expression levels of TaFH genes responded to stress stimuli, $1.5-\mathrm{kb}$ upstream promoter regions of $\mathrm{TaFH}$ genes were scanned for stress-related cis-regulatory elements using the Plant CARE online service. Eight hormone-responsive regulatory elements (ABRE, TGA-element, AuxRR-core, TGACG-motif,
CGTCA-motif, P-box, GARE-motif, and TCA-element) associated with ABA, auxin (IAA), methyl jasmonate (MeJA), gibberellin (GA), and salicylic acid (SA) responses were identified in the promoter region of TaFHs (Fig. 4) Additionally, five stress-responsive regulatory elements (TC-rich repeats, MBS, ARE, GC-motif, and LTR) associated with defense/stress and low-temperature responses were identified in the $\mathrm{TaFH}$ promoter regions. Different types and numbers of regulatory elements were present in the distinct TaFH promoters. This implied that TaFH genes are potentially associated with the response to various stress and hormone treatments by mediating different regulatory mechanisms.

\section{Tissue/organ-specific expression profiles of $\mathrm{TaFH}$ genes}

We explored the expression of TaFH genes in different tissues of TGMS wheat line BS366, including the root, stem, leaf, pistil, stamen, and glume. All the TaFH genes were constitutively expressed in all the tissues. The expression of TaFH1 in pistils was the highest, whereas that in stamens was about 45 folds more than in the roots (Fig. 5). The expression of TaFH6, TaFH3, and TaFH8 in pistils was markedly higher than those in other tissues, about 20, 3 and 1.2 folds more than in roots. The expression of TaFH4 and TaFH5 in stamens was the highest, about 30 and 120 folds more than that 


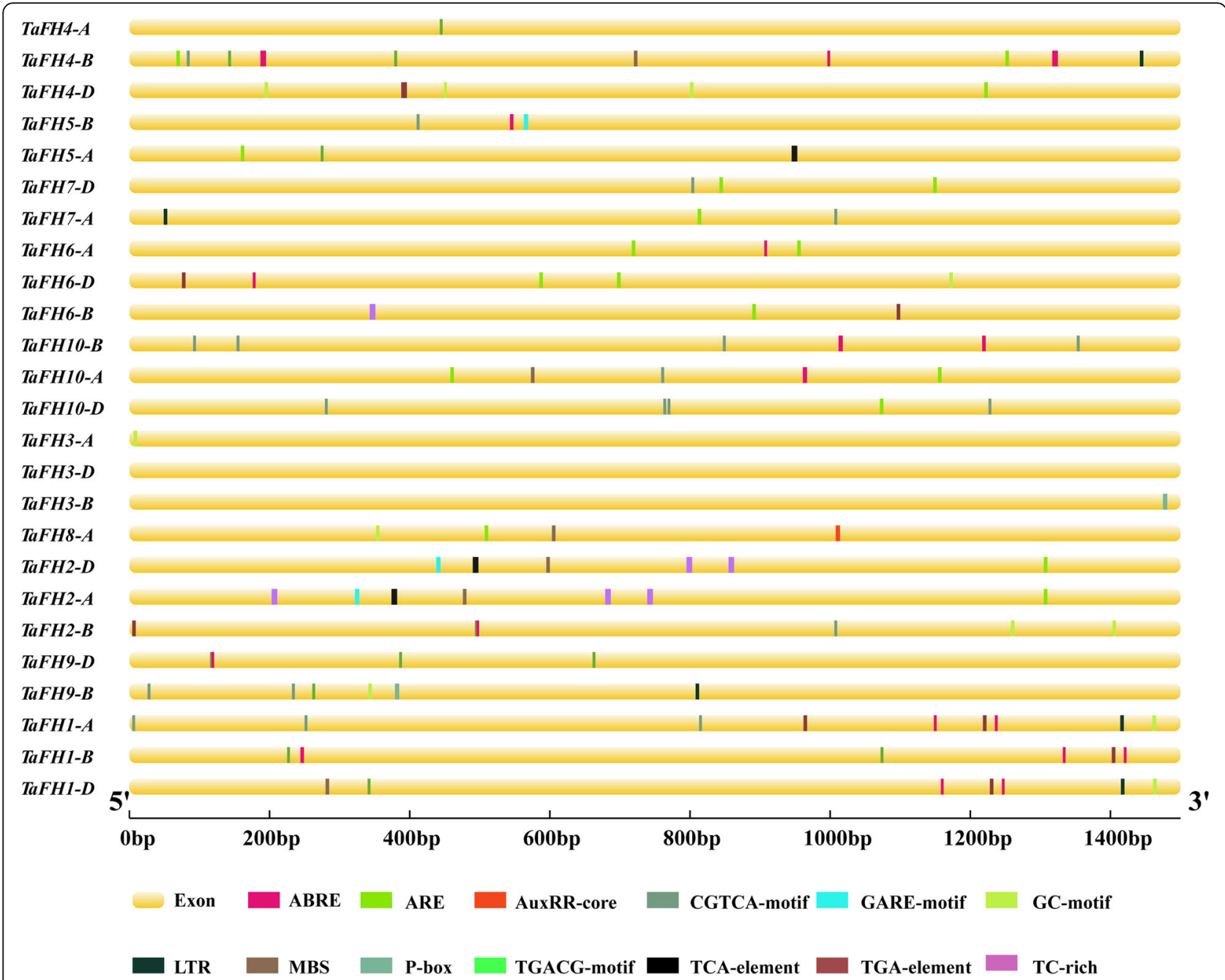

Fig. 4 Analysis of specific cis-elements in promoters of TaFHs. The $1.5 \mathrm{k}$-bp promoter sequences of corresponding TaFH genes were used to analyze specific hormone-related cis-elements and stress-responsive regulatory elements, which are color-coded. ABRE: abscisic acid responsive element; ARE: antioxidant response element mediating transcriptional activation of genes exposed to oxidative stress; AuxRR-core: auxinresponsive element; CGTCA-motif and TGACG-motif: MeJA-responsive element; GARE-motif and P-box: gibberellin-responsive element; GC-motif: enhancer-like element involved in anoxic specific inducibility; LTR: low-temperature responsive element; MBS: drought-inducible element; TCAelement: salicylic acid responsive element; TGA-element: auxin responsive element; TC-rich repeat: defense and stress responsive element

in roots, implying that TaFH1, TaFH4 and TaFH5, were potentially linked to anther development. Also, TaFH2 expression in leaves was the highest, nearly 9 folds higher than in the roots, but relatively lower in the corresponding tissues of flower organs. This suggested that TaFH 2 could be mainly related to the development of leaves. The expression of TaFH7 in glume was the highest, nearly 16 folds higher than that in the roots. The expression levels of TaFH9 and TaFH1O in glumes were higher than the root, stem, leaf, pistil and stamen, about 9 and 20 folds than in roots, respectively. Thus, we speculated that TaFH7, TaFH9, and TaFH1O could be related to the opening and closing of glumes. Further analysis revealed that 10 members of the wheat formin gene family were mainly associated with the development of the above-ground tissues of BS366, mostly the floral organs. In particular, they contributed to the development of anthers and glumes, and other metabolic processes in TGMS wheat.

\section{Analysis of TaFH genes family expression patterns in fertility sensitive stages under different fertility conditions in BS366}

Based on previous reports, the fertility sensitive stage of BS366 was the meiosis stage of microspore mother cells $[10,16]$. Herein, we selected three key meiotic stages (1, 2, 3) for expression pattern analysis. The role of the wheat formin gene family in pollen fertility was further elucidated by analyzing the expression of TaFHs in the three stages of stamen development in BS366 under 

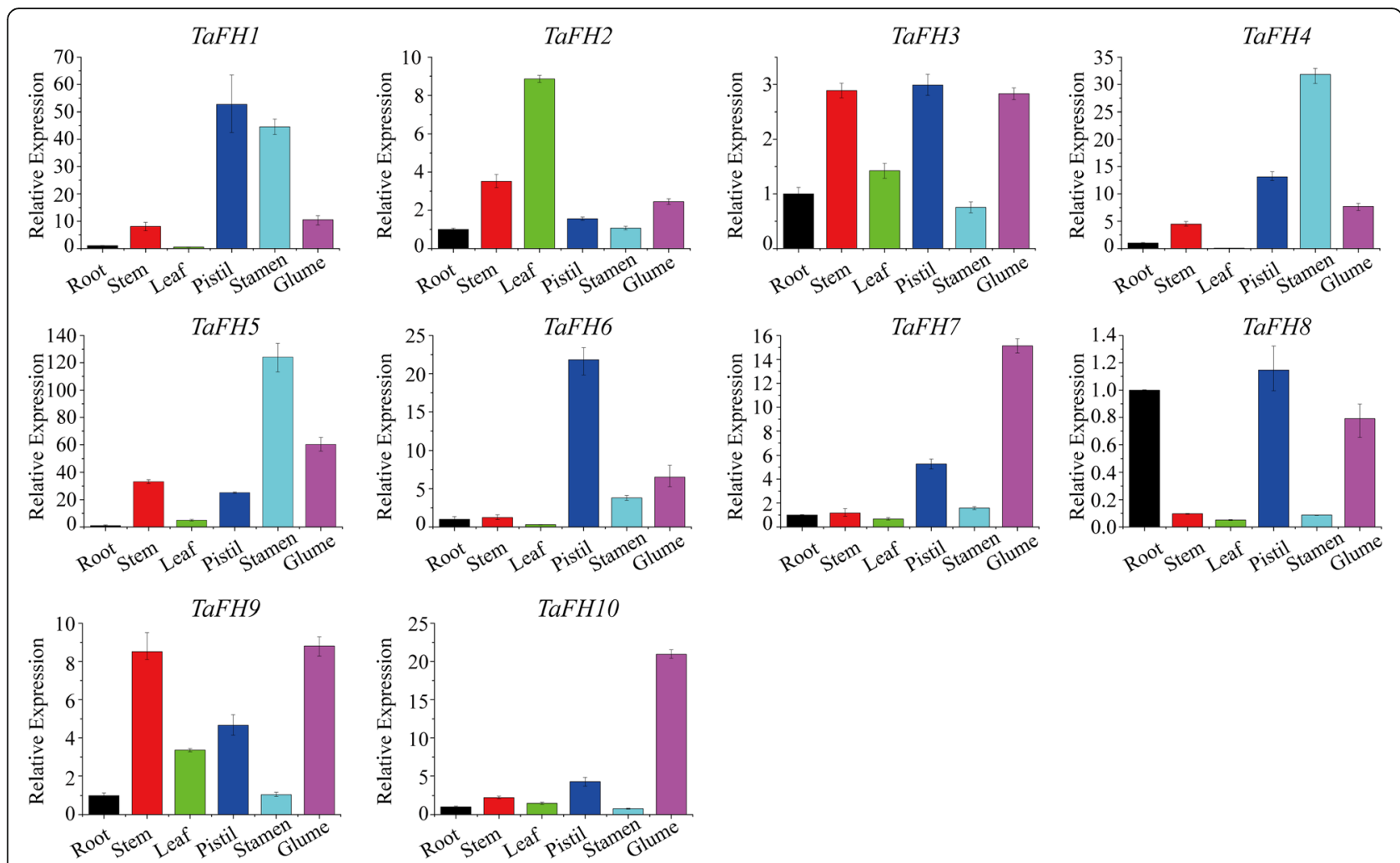

Fig. 5 The tissue expression patterns of the formin gene family in wheat, including root, stem, leaf, pistil, stamen, and glume. Different colors denoted different tissues; black for root, red for the stem, green for leaf, mazarine for pistil, wathet for stamen, purple for glume. The expression in root was set as 1. The error bars represent the standard error of the mean

different fertility environments (fertile and sterile conditions). The expression levels of TaFH2 and TaFH4 in the corresponding three stages of stamen development under sterile conditions were lower than those under fertile conditions (Fig. 6). TaFH1 expression was higher at the early stage (stage 1) under sterile conditions. The expression of TaFH1, TaFH3, TaFH5, and TaFH1O were significantly higher at stage1, but stage 2 under sterile conditions. On the other hand, TaFH6 expression was higher at the early stages of stamen development (stage 1 and 2) under sterile conditions. TaFH7 and TaFH8 showed no obvious expression changes at the three stages under both fertility and sterile conditions. However, TaFH9 expression was lower under sterility conditions than in fertility conditions at stage 1 and 2 . These results implied a potential association of TaFH genes with stamen development and fertility transformation via particular pathways.

\section{Distribution of cytoskeleton in meiosis of microsporocytes} Compelling evidence shows that the pollen cell cytoskeleton of BS366 is abnormal at low temperature, and the formin gene is highly critical in the development of the pollen cell cytoskeleton $[15,16]$. In the present study, to establish any change in the cytoskeleton of the TGMS
366 in the fertility and sterility environment, the pollen cell was evaluated in three key meiotic stages. We found abnormal cytoskeleton distribution in male-sterile wheat pollen cells (Fig. 7). The pollen cell shrank, and the cytoskeleton was loose and disarranged in stage 1 . The cell plate was absent, and the microtubule and microfilaments nonpolar terminus were vague in stage 2 . In stage 3 , the microtubule and microfilaments disappeared. Collectively, these findings suggested that differential expression of TaFH genes might cause the abnormal pollen cytoskeleton of BS366 under low temperatures.

\section{Expression profiles of $\mathrm{TaFH}$ genes under different exogenous phytohormone treatments and abiotic stress} Abscisic acid (ABA), gibberellin (GA), indole-3-acetic acid (IAA), methyl jasmonate (MeJA), and salicylic acid (SA) are the main plant growth regulators that exert critical functions in plant growth and metabolism. By exploring the cis-element of $\mathrm{TaFH}$, we found that $\mathrm{TaFH}$ had different types and numbers of regulatory elements. To affirm the function of $T a F H$, we conducted a qRTPCR assay to examine the expression pattern of the formin genes in the leaves of BS366 following different hormone treatments (Fig. 8). 


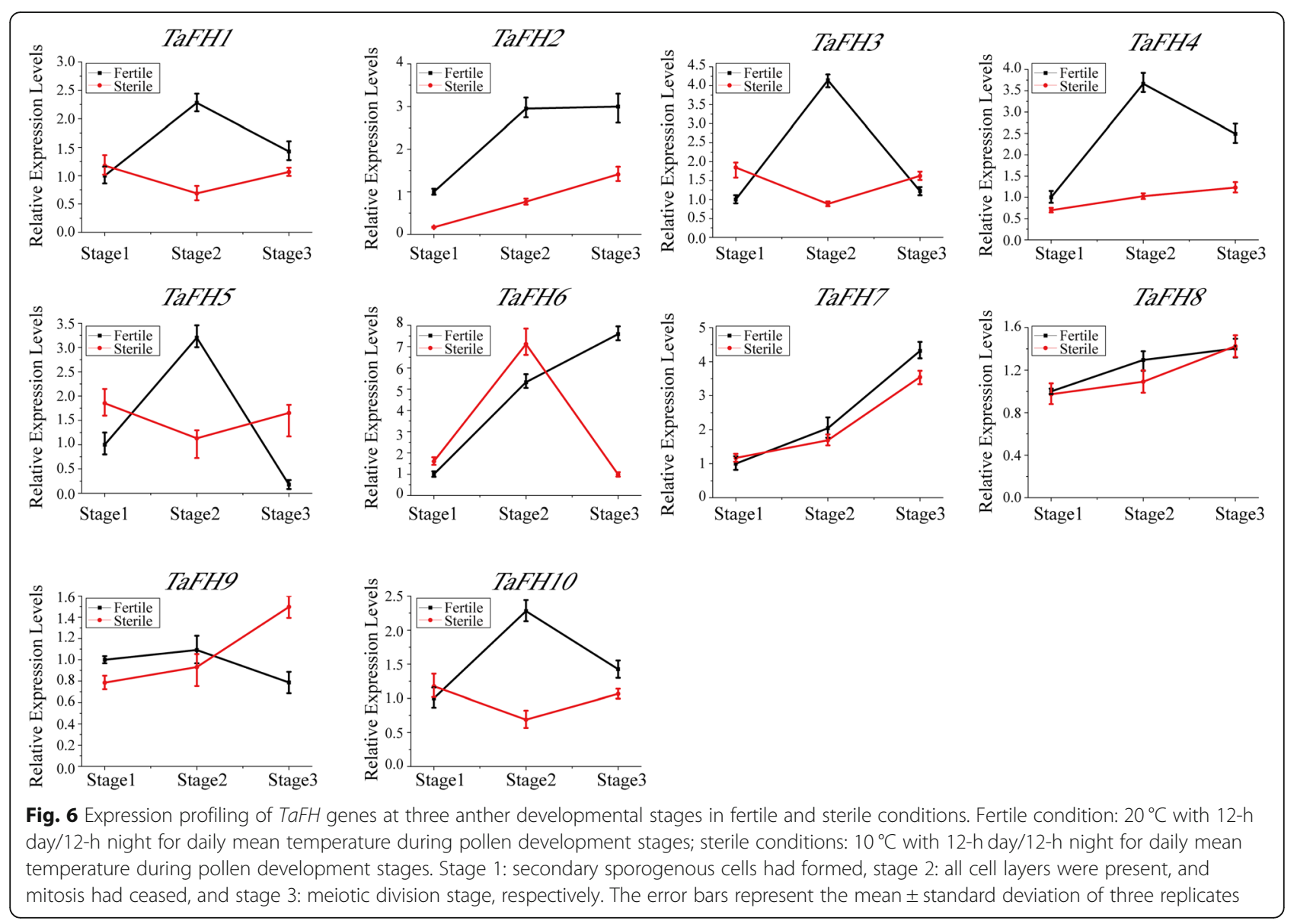

According to the results, all the formin genes slightly responded to the ABA, GA, IAA, MeJA, and SA treatments. Specifically, TaFH3, TaFH4, TaFH5, TaFH7, TaFH8, TaFH9, and TaFH10 were no obvious expressed changing under abscisic acid-induced stress, the expression of TaFH1 and TaFH6 was initially inhibited but later rose and peaked at $8 \mathrm{~h}$, after which it began to show a wave-like decreasing trend and then rose again. Under auxin (IAA) stress, TaFH1, TaFH2, TaFH3, TaFH4, TaFH5, TaFH6, TaFH7, TaFH8, and TaFH1O were initially overexpressed, but their expression later decreased. The expression of TaFH1, TaFH2, and TaFH 8 peaked at $4 \mathrm{~h}$, whereas TaFH9 expression was impeded and then increased, and later peaked at $12 \mathrm{~h}$. The expression of TaFH2, TaFH3, TaFH4, TaFH5, TaFH6, TaFH7, TaFH8, TaFH9, and TaFH10 demonstrated a certain upward trend under gibberellin stress. The expressions of TaFH 2 and TaFH1O rose significantly at $2 \mathrm{~h}$ after gibberellin stress, then decreased and increased again. TaFH3, TaFH4, TaFH5, TaFH6, TaFH7, TaFH8, and TaFH9 showed a wave-like increasing trend, then decreased and rose again. The expression of TaFH4, TaFH5, TaFH6, TaFH7, and TaFH8 peaked at $24 \mathrm{~h}$, whereas that of TaFH9 peaked at $4 \mathrm{~h}$. Under methyl jasmonate stress, TaFH1 expression was inhibited but later increased at 12-24 h. The expression of TaFH2, TaFH3, TaFH4, and TaFH5 was inhibited within $24 \mathrm{~h}$, and that of TaFH6 and TaFH1O showed an upward and downward trend, then peaked at $24 \mathrm{~h}$. Further, the expression of TaFH7 was inhibited at $2 \mathrm{~h}$, then increased and later decreased. TaFH8 expression peaked at $8 \mathrm{~h}$, increased at $2 \mathrm{~h}$, but then decreased, increased, and then decreased again at $12 \mathrm{~h}$; the relative expression peaked at $2 \mathrm{~h}$. The expression of TaFH9 was enhanced, then inhibited, and peaked at $8 \mathrm{~h}$. Under salicylic acid stress, the relative expression of TaFH2, TaFH5, TaFH7, TaFH8, TaFH9, and TaFH1O was higher at $2 \mathrm{~h}$ compared to $0 \mathrm{~h}$. TaFH2 expression peaked at $2 \mathrm{~h}$, while TaFH5 peaked at $8 \mathrm{~h}$. The expression of TaFH7, TaFH8, TaFH9 and TaFH1O all peaked at $4 \mathrm{~h}$. Besides, the relative expression of TaFH1, TaFH3 and TaFH6 decreased at $2 \mathrm{~h}$, whereas that of TaFH1 decreased at $8 \mathrm{~h}$. Also, the expression of TaFH3, TaFH4, and TaFH6 peaked at $4 \mathrm{~h}$. These results demonstrated that formin genes might be central to hormone regulation in the BS366 wheat.

To evaluate the response mechanism of the formin gene family to cold stress, drought, and high salt, we employed qRT-PCR to analyze the expression pattern of 


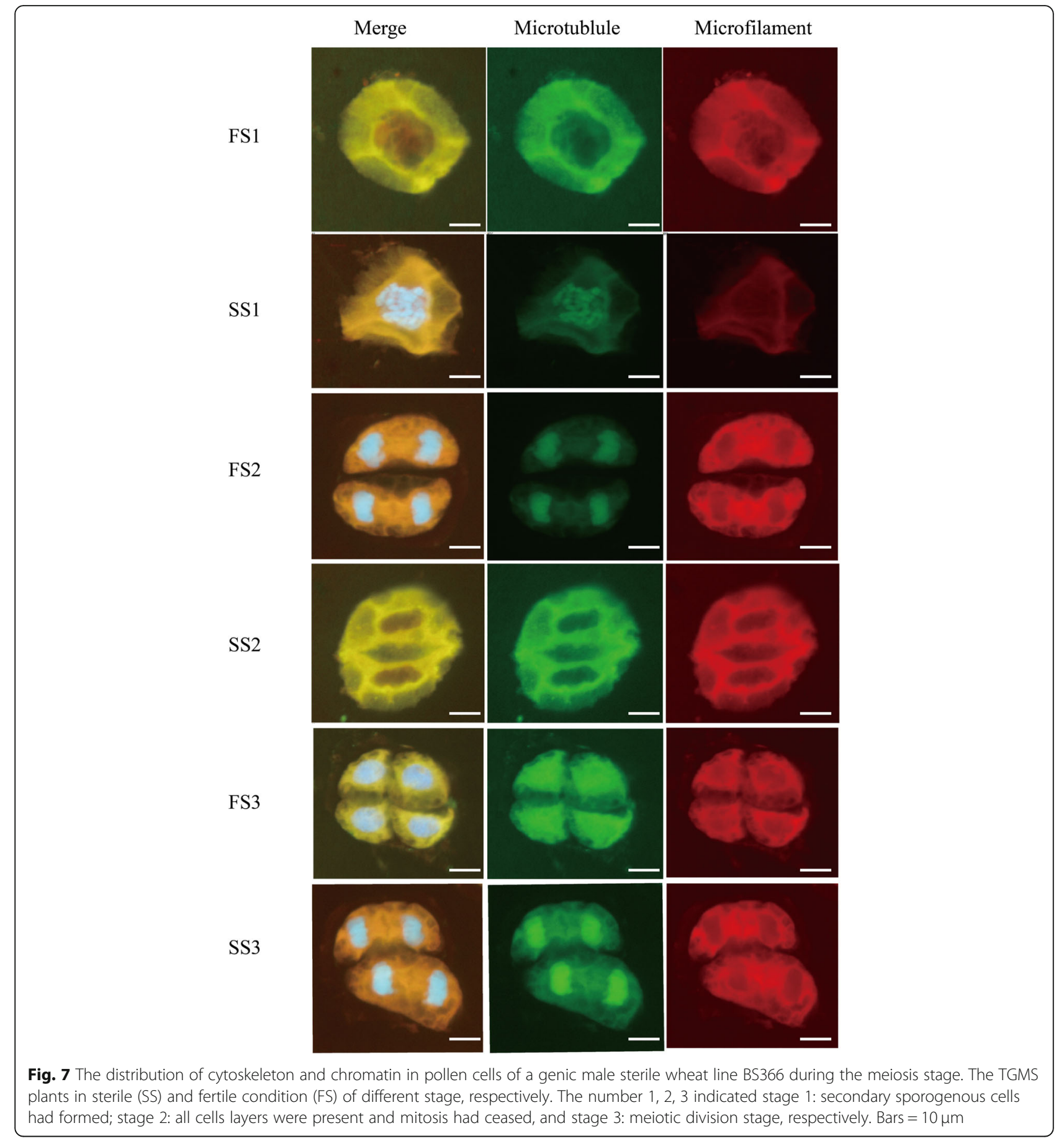

the formin genes in the leaves of BS366 under low temperature $\left(10^{\circ} \mathrm{C}\right)$, salt $(\mathrm{NaCl})$, and drought stress (PEG) treatments.

Initially, the expression of TaFH2, TaFH3, TaFH4, TaFH5, TaFH8, TaFH9, and TaFH10 was inhibited under cold stimulation, but later rose. The expression of most TaFH genes peaked at $12 \mathrm{~h}$; however, the expression of TaFH1, TaFH6, and TaFH7 rose and then decreased post-cold stimulation. High salt stress impeded the expression of TaFH1, TaFH4, TaFH7, TaFH8, TaFH9, and TaFH10, whereas the expression of TaFH2, TaFH3, TaFH5, and TaFH6 showed an opposite trend. Under abiotic drought stress, except for TaFH7 and TaFH8, the expression of TaFH2, TaFH3, and TaFH9 peaked at $8 \mathrm{~h}$ and then decreased. These results indicate that TaFH genes might respond to different plant hormones and participate in a complex regulatory network in thermo-sensitive male sterile line BS366. 


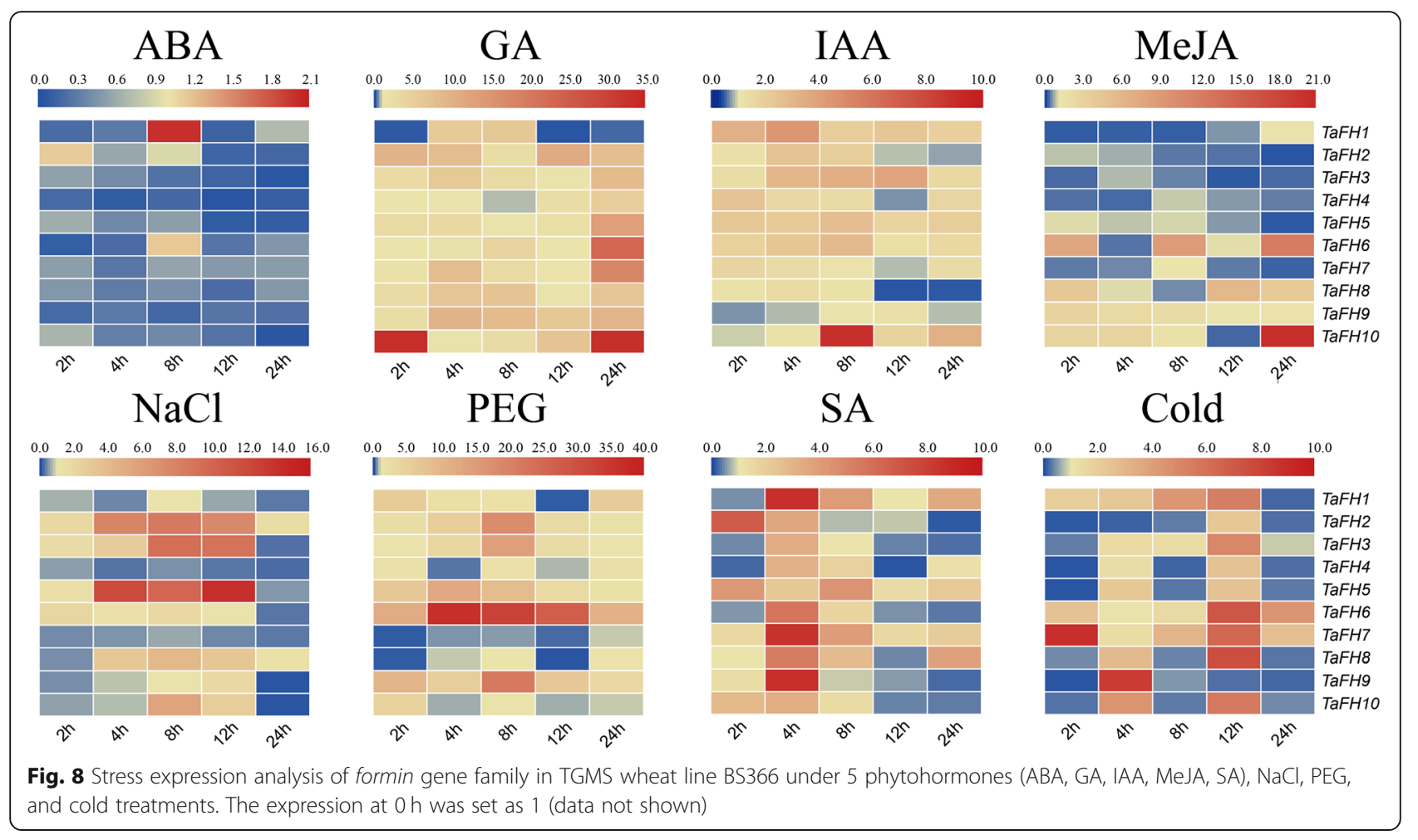

\section{Discussion}

The relationship between formin genes and cytoskeleton in wheat

Formins initiate actin assembly and control key cytoskeleton genes. They regulate the nucleation of actin and mediate profilin and Arp $2 / 3$ during cell division. Also, they participate in vegetative and reproductive plant growth [21]. Previously, [15] found abnormal cytoskeleton formation in a TGMS line under sterile environment. In this study, we examined the cytoskeleton of the pollen-sensitive stages in BS366 using scanning electron microscopy and found aberrant nuclear division in the pollen cells. Real-time quantification analysis of the formin genes revealed different expression of TaFH1, TaFH2, TaFH3, TaFH4, TaFH5, TaFH6, TaFH9 and TaFH1O under sterile and fertile conditions at these stages. Therefore, we concluded that the formin genes TaFH1, TaFH2, TaFH3, TaFH4, TaFH5, TaFH6, TaFH9, and TaFH1O might affect the cytoskeleton, resulting in abnormalities during meiosis and thus male sterility in TGMS BS366.

\section{Effect of microRNA regulation via formin genes on the fertility of TGMS line}

MiRNAs are endogenous, small, non-coding RNAs, exerting critical roles in the regulation of hormone signaling pathways and cellular functions during developmental processes [22]. Of note, miRNAs participate in the regulation of male fertility in crops [23]. Particularly,
miR167 plays a dominant role in plant development. In Arabidopsis, miR167 directly targets ARF6 and ARF8, which regulates jasmonate biosynthesis by impeding downstream genes, thereby affecting pollen development. In rice, miR167 potentially targets $A R F 6,8,12,17$, and 25 genes. Notably, ARF8 can interact with OsGH32. Besides, OsmiR167 responds to exogenous auxin via its target gene OsARF8. Abnormal OsGH3-2 expression induces morphological aberrations, including short plants and small panicles [24-26]. Studies have shown that TaemiR167a, TaemiR167b, and TaemiR167d are abnormally expressed in the sensitive stage of pollen in wheat line BS366 [27, 28]. TaemiR167a targeted AtARF6 and AtARF8 upon its translation into Arabidopsis, and influenced the synthesis of IAA and JA, thereby regulating the development of anthers [18].

In the present study, by analysis the relationship between the miRNA and formins, we found that taemiR167a targeted TaFH2-A, TaFH2-B, and TaFH2-D; taemiR167b targeted TaFH1-A, TaFH1-B, TaFH1-D, TaFH2-A, TaFH2-B, and TaFH2-D; whereas taemiR167c-5p targeted TaFH2-A, TaFH2-B, and TaFH2-D. As such, we suggested that taemiR167 might regulate the formin genes in pollensensitive stages and influence BS366 fertility; leading to abnormal anther dehiscence. Moreover, the gene FBA (fructose-1, 6-bisphosphate aldolase) was predicted to be the target of miR1120. Of note, miR1120 could be regulated by ABA under low-temperature treatment, which affected the expression of $F B A$, then regulated the sugar 
metabolism to mediate ATP generation [28]. In a previous report, the OsFH5 mutants were characterized by aberrant inflorescence. The cytological analysis demonstrated that OsFH5 mutants exhibited severe cell elongation defects and abnormal microtubule arrays [29]. Herein, miR1120 was predicted to target TaFH3 (Fig. 3), OsFH5, and TaFH3, clustered into group B (Fig. 1). Also, the cytoskeleton was abnormal during anther development. Thus, we speculated that miR1120 potentially regulated the energy synthesis and then impacted the pollen cell division and the fertility of the TGMS line BS366 under low-temperature treatment.

\section{Expression and functions of formin genes in response to abiotic stressors}

A previous study by [27] found abnormal levels of IAA during TGMS366 pollen development. Other reports have also revealed that MeJA, JA, and SA influence the pollen spill out, which results in the sterility of BS366 [30, 31]. In this study, we isolated the formin gene family, including 25 members from wheat (Table 1). The formin genes exhibited different expression levels in different BS366 tissues. For instance, the relative expression of TaFH4 and TaFH5 in stamen was higher compared to other tissues. Also, the relative expression of TaFH1, TaFH3, TaFH6, and TaFH8 in pistil was higher than in other tissues, suggesting that TaFH1, TaFH3, TaFH4, TaFH5, TaFH6, and TaFH8 potentially contribute to the sterility of BS366. Furthermore, the relative expression of TaFH1, TaFH2, TaFH3, TaFH4, TaFH5, TaFH6, TaFH9, and TaFH1O was different in the sensitive stage between the fertility and sterility conditions, implying that they might play a regulatory role in wheat fertility.

Cis-regulatory sequences, such as enhancers and promoters, influence development and physiology by regulating gene expression [32]. Cis-regulatory elements, functioning as important molecular switches, are associated with the regulation of gene transcription under external stimuli [33]. To comprehensively elucidate the functions of TaFH genes, we assessed cis-acting regulatory elements and expression profiles under various stresses. Eight responsive regulatory elements and five stress-responsive regulatory elements were identified in the TaFH promoter region. These findings demonstrated that the TaFH genes respond to stress and hormone treatment variations via different mechanisms. The TaFHs contained different types and numbers of cis-acting regulatory elements in each promoter region. These genes, therefore, were characterized by different regulatory functions that responded to variations in stress and hormone treatments. We also evaluated the expression profiles of TaFH genes in TGMS wheat line BS366 seedlings under different stress conditions and hormone treatments. The expression profile analysis revealed that
TaFH8 and TaFH10 had similarly structured proteins upregulated by the auxin response treatment. Similar expression profiles were shown in the $\mathrm{TaFH} 2$ following low-temperature treatment.

Moreover, the response of formin genes to the $\mathrm{ABA}, \mathrm{NaCl}$, and PEG implied that TaFHs might exert multiple functions in plants, including roles in abiotic stress resistance and pollen fertility. Although formin is a key regulatory gene in the cell cytoskeleton, exploring the mechanisms by which it responds to low temperature, affects the cytoskeleton, and defines pollen development of wheat would be vital to reveal the actual function of TaFH.

\section{Possible miRNA-TaFH interaction network in wheat}

In this study, we proposed a potential regulatory pathway of TaFH (Fig. 9). The functions of the TaFH gene family were established by multiple endogenous and exogenous factors, and the regulatory network was complex. Therefore, we speculated that these miRNAs of tae-miR167, tae-miR1120-3p, tae-miR9677, tae-miR9679, tae-miR444a, tae-miR172, tae-miR9781, taemiR9675 and tae-miR9661 could inhibit the expression of target TaFHs at the transcriptional or translational levels under low-temperature conditions. This regulation strategy could have contributed to the abnormal cytoskeleton and the meiosis abnormalities, resulting in abnormal pollen. Besides, TaFH genes are associated with the signaling pathway of MeJA, SA, IAA, ABA, and GA. In this study, we found that TaFH1 and TaFH2 were regulated by IAA, TaFH4 and TaFH6 could respond to MeJA, and TaFH3 and TaFH9 were mainly regulated by GA. Collectively, these findings suggested that TaFH1, TaFH2, TaFH3, and TaFH9 might play a crucial role in the regulation of temperatureinduced male sterility in wheat TGMS line BS366.

\section{Conclusions}

In this study, we systematically uncovered $\mathrm{TaFH}$ genes in the wheat genome. A total of $25 \mathrm{TaFHs}$ were identified, each containing a conserved $\mathrm{FH} 2$ domain. The chromosome locations, gene and protein structures, subcellular localization, phylogenetic relationships, TaemiR167, Tae-miR1120, Tae-miR172, and Tae-miR444 binding sites, and cis-elements were also characterized. The TaFH expression levels in different tissues demonstrate their potential role in flower development. The pollen cytoskeleton of BS366 was abnormal in the three stages under fertility and sterility conditions. Based on quantitative RT-PCR analysis, the tested TaFH genes were highly expressed in inflorescences and response to abiotic stressors. Collectively, these findings establish a foundation for further exploration of TaFH genes and provide novel insights into their biological functions. 


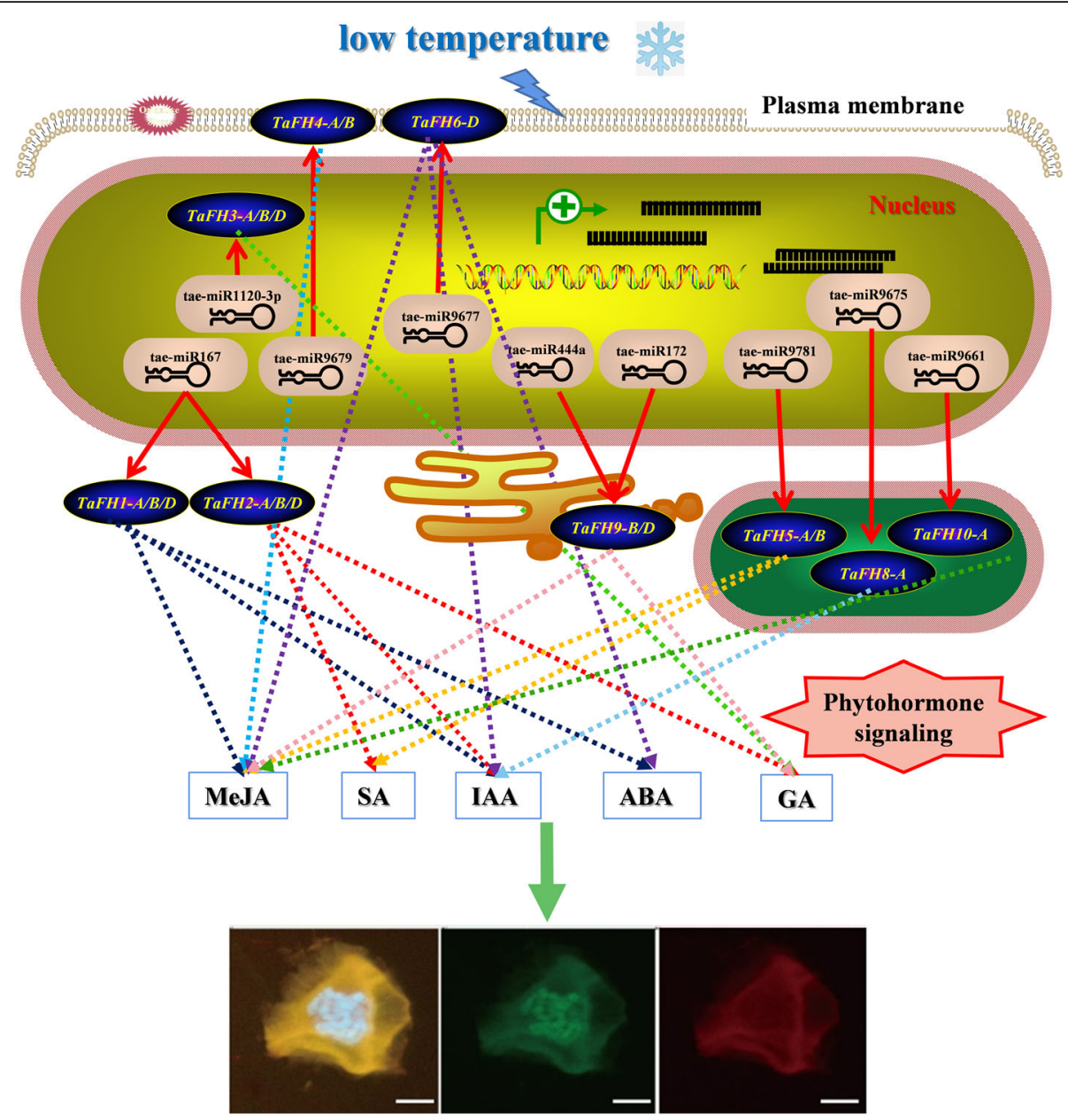

Fig. 9 Putative regulatory network of miRNAs and targeted formin genes in wheat. Arrows with dotted lines indicate the most sensitive responses to stresses of each TaFH gene. Arrows with a solid line indicate the interactions of tae-miRNAs and target TaFH genes negative control. Tae-miR167 may target TaFH1 and TaFH2 in the cytoplasm; tae-miR1120-3p may target TaFH3 in the nucleus; tae-miR9677 and tae-miR9679 may target TaFH6 and TaFH4 in the plasma membrane; tae-miR444a/tae-miR172 may targeted TaFH9 in endoplasmic reticulum; tae-miR9781, taemiR9675, and tae-miR9661 may target TaFH5, TaFH8, and TaFH10 in chloroplast

\section{Methods}

\section{Sources of sequence data}

The whole-genome sequences of Triticum aestivum $\mathrm{L}$. ( $T$. aestivum, Ta) were retrieved from the wheat genome Ensemble database (http://plant.ensembl.org, IWGSC RefSeqv1.1). To identify members of the formin genes family from $T$. aestivum, we adopted two methods: Firstly, the hidden Markov model (HMM) profile of the formin (accession number in Pfam: PF06507) was downloaded from the Pfam database (http://pfam.sanger.ac. $\mathrm{uk} /$ ) [34] and used as a query to search for candidate formin proteins in the T. aestivum proteome using HMMER3.0 [35]. Additionally, Arabidopsis formin protein sequences were adopted as queries to reveal the $T$. aestivum L. proteome via BLAST. Genes collected from the above two methods were integrated, and duplicates were eliminated manually. All protein sequences of the candidate formin genes were verified using domain analysis programs NCBI-CDD (https://www.ncbi.nlm.nih. gov/cdd/) and SMART (Simple Modular Architecture Research Tool; http://smart.emblheidelberg.de/), the gene structure with intro-exon boundaries of $\mathrm{TaFH}$ gene family using the Gene Structure Display Server (GSDS). Basic physical and chemical parameters of $\mathrm{TaFH}$ proteins, including amino acid length, theoretical isoelectric point, and molecular weight, were obtained from an online program, ProtParam (https://web.expasy.org/ protparam/).

\section{Phylogenetic analysis}

The formin coding sequences for Physcomitrella patens ( $P$. patens, Pp), Selaginella moellendorffii (S. moellendorffii, $\mathrm{Sm})$, Populus trichocarpa (P. trichocarpa, Pt), Sorghum bicolor (S. bicolor, Sb), Brachypodium distachyon (B. 
distachyon, Bd), Arabidopsis thaliana (A. thaliana, At), and Oryza sativa (O. sativa, Os) were acquired from the downloaded data (ftp://ftp.ensemblgenomes.org/). MUSCLE software (http://www.drive5.com) was applied for amino acid (aa) alignments. A phylogenetic tree was generated via the MEGA X software using the neighbor-joining (NJ) method and 1000 bootstrap tests and visualized using the online software tool EvolView [36].

\section{Homology modeling}

The 3D structure of wheat formin proteins was constructed by homology modeling method, which based on the resolved of homologous protein. The Phyre 2 server was been used to obtain the 3D structure and further visualized by PyMOLWin.

\section{Chromosomal distribution}

The location of each formin gene on the wheat chromosomes was mapped to IWGSC RefSeq v1.1 (cv. Chinese Spring) using blast programs (https://blast.ncbi.nlm.nih. gov/Blast.cgi). The centromere locations were retrieved from IWGSC (www.wheatgenome.org). Then, using the MapInspect tool (http://mapinspect.software.informer. $\mathrm{com} /$ ), their locations were drawn onto the physical map of each chromosome. The graphic of chromosomal distribution was refined using Adobe illustrator.

\section{Prediction of miRNA target genes}

MiRNAs and their targets in $\mathrm{TaFH}$ genes were predicted via a web-based psRNATarget server using default parameters [37]. They were then visualized using Cytoscape (version 3.7), as described previously [38].

\section{Identification of the cis-acting elements in the $\mathrm{TaFH}$ genes}

The cis-acting elements in the $1500 \mathrm{bp}$ upstream promoter regions of the identified TaFH genes were analyzed via PlantCARE (http://bioinformatics.psb.ugent. be/webtools/ plantcare/html/). All of these sequences were used to identify the cis-acting elements by the recently released $T$. aestivum genome database (http:// plant.ensembl.org/, IWGSC RefSeqv1.1).

\section{Plant materials, treatments, and sample collections}

The thermo-sensitive genic male sterile (TGMS) wheat line BS366 was planted in the experimental fields in Beijing (China, N39 $54^{\prime}$, E116 ${ }^{\circ} 18^{\prime}$ ) and Nanyang (Henan province, China, $\mathrm{N} 39^{\circ} 86^{\prime}, \mathrm{E} 116^{\circ} 25^{\prime}$ ) and managed conventionally. To analyze the roles of TaFH genes during pollen development in BS366, we sampled the anthers at three stages: stage 1 (secondary sporogenous cells had formed); stage 2 (all cell layers were present and mitosis had ceased); and stage 3 (meiotic division stage) [27].
For tissue-specific expression analysis, six tissues (root, stem, leaf, glume, stamen, pistil) from BS366 were collected in the field experiment station. Two-week-old wheat seedlings $\left(20^{\circ} \mathrm{C}, 12\right.$-h day/12-h night cycle) were used for abiotic stresses analysis, as per the methods described by Bai et al. [28]. In brief, we sprayed BS366 seedlings with $2 \mathrm{mM}$ SA, $100 \mathrm{mM}$ MeJA, $100 \mathrm{mM}$ GA, $50 \mathrm{mM}$ IAA, and $100 \mathrm{mM}$ ABA. For the high-salinity and drought treatments, the roots of wheat seedlings were soaked in $200 \mathrm{mM} \mathrm{NaCl}$ and PEG6000 (-0.5), and leaf tissues were collected from the seedlings $0,2,4,8$, 12 , and $24 \mathrm{~h}$ post-treatment. To evaluate the lowtemperature stress, 2 -week-old seedlings were incubated at $10{ }^{\circ} \mathrm{C}$, and leaf tissues were collected from the seedlings at $0,2,4,8,12$, and $24 \mathrm{~h}$ time points. All samples were frozen in liquid nitrogen immediately after collection. And a flowchart was provided in Fig. S4.

\section{Pollen cell and DNA staining}

Pollen cell staining was performed as described by Wang et al. [10] and Xu et al. [15]. Briefly, microfilaments and microtubules were respectively marked with tetramethylrhodamine isothiocyanate (TRITC)-phalloidin (Sigma, St. Louis, MO, USA) and anti- $\alpha$-tubulin (mouse IgG monoclonal anti- $\alpha$-tubulin, T-9026; Sigma) in pollen cells. DNA staining was conducted using 4', 6-diamidino-2-phenylindole (DAPI) for counterstaining as described by $\mathrm{Xu}$ et al. [15]. After examining the preparations, images were captured using a laser scanning confocal microscope (Nikon A1R, Tokyo, Japan).

\section{Gene expression assay/analysis}

For expression analysis of TaFH genes, total RNA was isolated from wheat tissues using TRIzol reagent (Invitrogen, USA) following the manufactures' instructions. First-strand cDNA synthesis was performed using a PrimeScriptTM RT Reagent kit with gDNA Eraser (TaKaRa, Japan). Next, qRT-RCR assay was conducted on an Eco Real-time PCR system (Illumina, USA) using SYBR $^{\bullet}$ Permix Ex TaqTM (TaKaRa, Japan), as described previously [31].

Relative expression levels of TaFH genes were determined using the comparative threshold cycle method $2^{-}{ }_{\Delta} \mathrm{CT}$ [39]. Wheat $18 S$ gene served as the reference control. All the qRT-PCR reactions were conducted in three biological replicates. The primer used for qRTPCR were conserved for all three homologues and designed using Primer premier 5.0 program and are listed in Table S2.

\section{Abbreviations}

ABA: Abscisic acid; GA: Gibberellin; IAA: Indole-3-acetic acid; MeJA: Methyl jasmonate; TGMS: Thermo-sensitive genic male sterile 


\section{Supplementary Information}

The online version contains supplementary material available at https:/doi. org/10.1186/s12864-021-07878-7.

Additional file 1: Figure S1. Phylogenetic relationship and motif structure of wheat formin proteins. The phylogenetic tree of TaFH proteins constructed from a complete alignment of 25 wheat formin proteins using MEGA 6.0 by the neighbor-joining method with 1000 bootstrap replicates. Domain distribution of $\mathrm{TaFH}$ proteins were investigated using the MEME web server.

Additional file 2: Figure S2. Exon-intron structures of TaFH genes. Exons are represented by green boxes and introns by blank lines.

Additional file 3: Figure S3. Three-dimensional structure of representative $\mathrm{TaFH}$ protein.

Additional file 4: Figure S4. The flowchart of TaFH gene family with the treatment of BS366.

Additional file 5: Table S1. The interaction of miRNAs with TaFH genes. Table S2. The primers of the qRT-RCR in TaFHs.

\section{Acknowledgements}

We are grateful to the reviewers for their helpful suggestions. We thank editors very much for their efficient works.

\section{Authors' contributions}

WD, $L Z$ and $C Z$ designed the study. WD, ZL and JB performed the experiments, analyzed the data, and drafted the manuscript. JS and FL assisted with bioinformatics analysis and aided in writing the manuscript. SY, $\mathrm{YL}, \mathrm{TZ}$ and $\mathrm{FZ}$ aided in performing the experiments. The author(s) read and approved the final manuscript.

\section{Funding}

This research was financially supported by the Science and Technology Plan of Beijing (Z191100004019014), National Natural Science Foundation of China (31872881), Outstanding Scientist Cultivation Program of BAAFS

(JKZX201907) and the Beijing Excellent Talents Project.

\section{Availability of data and materials}

The data sets supporting the results of this article are included within the article and its additional files.

All the links of datasets used in the study:

The wheat genome Ensemble database (http://plant.ensembl.org/).

The Pfam database (http://pfam.sanger.acuk/).

NCBI-CDD (https://www.ncbi.nlm.nih.gov/cdd/).

SMART (http://smart.emblheidelberg.de/).

ProtParam (https://web.expasy.org/)

The formin coding sequences for $P$. patens, S. moellendorffii, P. trichocarpa, S. bicolor, B. distachyon, A.thaliana, and O. sativa were acquired from the downloaded data (ftp://ftp.ensemblgenomes.org).

MUSCLE software (http://www.drive5.com)

IWGSC RefSeq v1.1 (cv. Chinese Spring) (https://blast.ncbi.nlm.nih.gov/Blast.cgi). The location of each formin gene on the wheat chromosomes was mapped to IWGSC RefSeq v1.1 (cv. Chinese_Spring) using blast programs (https:// blast.ncbi.nlm.nih.gov/Blast.cgi).

The centromere locations were retrieved from IWGSC (www.wheatgenome.org). The Maplnspect tool (http://mapinspect.software.informer.com/). PlantCARE (http://bioinformatics.psb.ugent. be/webtools/ plantcare/html/).

\section{Declarations}

\section{Ethics approval and consent to participate}

TGMS wheat line BS366 seeds were provided by Beijing Engineering and Technique Research Center for Hybrid Wheat. The experimental research and field studies on plants, including the collection of plant material, were complied with relevant institutional, national, and international guidelines and legislation.

\section{Consent for publication}

Not applicable.

\section{Competing interests}

The authors declare that they have no competing interests.

\section{Author details}

${ }^{1}$ Beijing Engineering and Technique Research Center for Hybrid Wheat, Beijing Academy of Agriculture and Forestry Sciences, Beijing 100097, China. ${ }^{2}$ The Municipal Key Laboratory of Molecular Genetic of Hybrid Wheat, Beijing 100097, China. ${ }^{3}$ College of Life Science, Capital Normal University, Beijing 100048, China.

Received: 8 April 2021 Accepted: 1 July 2021

Published online: 24 July 2021

\section{References}

1. Kumagai F, Hasezawa S. Dynamic organization of microtubules and microfilaments during cell cycle progression in higher plant cells. Plant Biol. 2001;3(1):4-16. https://doi.org/10.1055/s-2001-11749.

2. Evangelista $M$, Zigmond $S$, Boone C. Formins: signaling effectors for assembly and polarization of actin filaments. J Cell Sci. 2003;116(13):260311. https://doi.org/10.1242/jcs.00611.

3. Lan Y, Liu X, Fu Y, Huang S. Arabidopsis class I formins control membraneoriginated actin polymerization at pollen tube tips. PLoS Genet. 2018;14(11): e1007789. https://doi.org/10.1371/journal.pgen.1007789.

4. Cheung AY, Wu HM. Overexpression of an Arabidopsis formin stimulates supernumerary actin cable formation from pollen tube cell membrane. Plant Cell. 2004;16(1):257-69. https://doi.org/10.1105/tpc.016550.

5. Favery B, Chelysheva LA, Lebris M, Jammes F, Marmagne A, Janice DAE, et al. Arabidopsis formin AtFH6 is a plasma membrane-associated protein upregulated in giant cells induced by parasitic nematodes. Plant Cell. 2004; 16(9):2529-40. https://doi.org/10.1105/tpc.104.024372.

6. Yi KX, Guo CQ, Chen D, Zhao BB, Yang B, Ren HY. Cloning and functional characterization of a formin-like protein (AtFH8) from Arabidopsis. Plant Physiol. 2005;138(2):1071-82. https://doi.org/10.1104/pp.104.055665.

7. Deeks MJ, Cvrcková F, Machesky LM, Mikitová V, Ketelaar T, Zársky V, et al. Arabidopsis group le formins localize to specific cell membrane domains, interact with actin-binding proteins and cause defects in cell expansion upon aberrant expression. New Phytol. 2005;168(3):529-40. https://doi.org/1 0.1111/j.1469-8137.2005.01582.x.

8. Guo CQ, Ren HY. Formins: bringing new insights to the organization of actin cytoskeleton. Chin Sci Bull. 2006;51(24):2937-43. https://doi.org/10.1 007/s11434-006-2214-X.

9. Zheng YY, Xin HB, Lin JX, Liu CM, Huang SJ. An Arabidopsis class II formin, AtFH19, nucleates actin assembly, binds to the barbed end of actin filaments, and antagonizes the effect of AtFH1 on actin dynamics. J Integr Plant Biol. 2012;54(10):800-13. https://doi.org/10.1111/j.17447909.2012.01160.x.

10. Wang YK, Bai JF, Wang P, Duan WJ, Yuan SH, Zhang FT, et al. Comparative transcriptome analysis identifies genes involved in the regulation of the pollen cytoskeleton in a genic male sterile wheat line. Plant Growth Regul. 2018;86(1):133-47. https://doi.org/10.1007/s10725-018-0416-2.

11. Virmani SS, llyas-Ahmed M. Environment-sensitive genic male sterility (EGMS) in crops. Adv Agron. 2001;72(01):139-95. https://doi.org/10.1016/ S0065-2113(01)72013-5.

12. Sj K, Cho KH, Choi YJ, Baek WK, Kim S, Suh HS. Cytological observation of two environmental genic male-sterile lines of rice. Mol Cell. 2001;12(3):403-6.

13. Li YF, Zhao CP, Zhang FT, Sun H, Sun DF. Fertility alteration in the photothermo-sensitive male sterile line BS20 of wheat (Triticum aestivum L.). Euphytica. 2006;151(2):207-13. https://doi.org/10.1007/s10681-006-9141-4.

14. Xing QH, Ru ZG, Li J, Zhou CJ, Jin DM, Sun Y, et al. Cloning a second form of adenine phosphoribosyl transferase gene (TaAPT2) from wheat and analysis of its association with thermo-sensitive genic male sterility (TGMS) Plant Sci. 2005;169(1):37-45. https://doi.org/10.1016/j.plantsci.2005.02.028.

15. Xu CG, Liu ZT, Zhang LP, Zhao CP, Yuan SH, Zhang FT. Organization of actin cytoskeleton during meiosis I in a wheat thermo-sensitive genic male sterile line. Protoplasma. 2013;250(1):415-22. https://doi.org/10.1 007/s00709-012-0386-6.

16. Tang ZH, Zhang LP, Yang D, Zhao CP, Zheng YL. Cold stress contributes to aberrant cytokinesis during male meiosis I in a wheat thermosensitive genic male sterile line. Plant Cell Environ. 2011;34(3):389-405. https://doi.org/1 0.1111/j.1365-3040.2010.02250.x. 
17. Ambros V. MicroRNAs: tiny regulators with great potential. Cell. 2001;107(7): 823-6. https://doi.org/10.1016/S0092-8674(01)00616-X.

18. Wang YK, Duan WJ, Bai JF, Wang P, Yuan SH, Zhao CP, et al. Constitutive expression of a wheat microRNA, TaemiR167a, confers male sterility in transgenic Arabidopsis. Plant Growth Regul. 2019;88(7):227-39. https://doi. org/10.1007/s10725-019-00503-4

19. Liu P, Liu J, Dong HX, Sun JQ. Functional regulation of $Q$ by microRNA172 and transcriptional co-repressor TOPLESS in controlling bread wheat spikelet density. Plant Biotechnol J. 2018;16(2):495-506. https://doi.org/1 $0.1111 /$ pbi.12790.

20. Gao S, Guo CJ, Zhang YS, Zhang FF, Du XM, Gu JT, et al. Wheat microRNA member TaMIR444a is nitrogen deprivation-responsive and involves plant adaptation to the nitrogen-starvation stress. Plant Mol Biol Report. 2016; 34(5):931-46. https://doi.org/10.1007/s11105-016-0973-3.

21. Romero S, Le-Clainche C, Didry D, Egile C, Pantaloni D, Carlier M-F. Formin is a processive motor that requires profilin to accelerate actin assembly and associated ATP hydrolysis. Cell. 2004;119(3):419-29. https://doi.org/10.1016/j. cell.2004.09.039.

22. Gupta OP, Permar V, Koundal V, Singh UD, Praveen S. MicroRNA regulated defense responses in Triticum aestivum L. during Puccinia graminis f.sp. tritici infection. Mol Biol Rep. 2012;39(2):817-24. https://doi.org/10.1007/s11033011-0803-5.

23. Li ZF, Zhang YC, Chen YQ. MiRNAs and IncRNAs in reproductive development. Plant Sci. 2015;238:46-52. https://doi.org/10.1016/j.plantsci.201 5.05.017.

24. Liu H, Jia SH, Shen DF, Liu J, Li J, Zhao HP, et al. Four AUXIN RESPONSE FACTOR genes downregulated by microRNA167 are associated with growth and development in Oryza sativa. Funct Plant Biol. 2012;39(9):736-44. https://doi.org/10.1071/FP12106.

25. Yang JH, Han SJ, Yoon EK, Lee WS. Evidence of an auxin signal pathway, microRNA167-ARF8-GH3, and its response to exogenous auxin in cultured rice cells. Nucleic Acids Res. 2006;34(6):1892-9. https://doi.org/10.1093/nar/ gkl118.

26. Du H, Wu N, Fu J, Wang SP, Li XH, Xiao JH, et al. A GH3 family member OsGH3-2 modulates auxin and abscisic acid levels and differentially affects drought and cold tolerance in rice. J Exp Bot. 2012;63(18):6467-80. https:// doi.org/10.1093/jxb/ers300.

27. Tang ZH, Zhang LP, Xu CG, Yuan SH, Zhang FT, Zheng YL, et al. Uncovering small RNA-mediated responses to cold stress in a wheat thermosensitive genic male-sterile line by deep sequencing. Plant Physiol. 2012;159(2):72138. https://doi.org/10.1104/pp.112.196048.

28. Bai JF, Wang YK, Wang P, Duan WJ, Yuan SH, Sun H, et al. Uncovering male fertility transition responsive miRNA in a wheat photo-thermosensitive genic male sterile line by deep sequencing and degradome analysis. Front Plant Sci. 2017;8:1370. https://doi.org/10.3389/fpls.2017.01370

29. Zhang Z, Zhang Y, Tan HX, Wang Y, Li G, Liang WQ, et al. Rice morphology determinant encodes the type II formin $\mathrm{FH} 5$ and regulates rice morphogenesis. Plant Cell. 2011;23(2):681-700. https://doi.org/10.1105/ tpc.110.081349.

30. Wang YK, Qiao LY, Bai JF, Wang P, Duan WJ, Yuan SH, et al. Genome-wide characterization of JASMONATE-ZIM DOMAIN transcription repressors in wheat (Triticum aestivum L.). BMC Genomics. 2017;18(1):152.

31. Bai JF, Wang YK, Wang P, Yuan SH, Gao JG, Duan WJ, et al. Genome-wide identification and analysis of the COI gene family in wheat (Triticum aestivum L.). BMC Genomics. 2018;19(1):754.

32. Wittkopp PJ, Kalay G. Cis-regulatory elements: molecular mechanisms and evolutionary processes underlying divergence. Nat Rev Genet. 2011;13(1): 59-69. https://doi.org/10.1038/nrg3095.

33. Nakashima K, Ito Y, Yamaguchi-Shinozaki K. Transcriptional regulatory networks in response to abiotic stresses in Arabidopsis and grasses1. Plant Physiol. 2009;149(1):88-95. https://doi.org/10.1104/pp.108.129791.

34. Finn RD, Coggill P, Eberhardt RY, Eddy SR, Mistry J, Mitchell AL, et al. The Pfam protein families database: towards a more sustainable future. Nucleic Acids Res. 2016;44(D1):D279-85. https://doi.org/10.1093/nar/gkv1344.

35. Eddy SR. A probabilistic model of local sequence alignment that simplifies statistical significance estimation. PLoS Comput Biol. 2008;4(5):e1000069. https://doi.org/10.1371/journal.pcbi.1000069.

36. He ZL, Zhang HK, Gao SH, Lercher MJ, Chen WH, Hu SN. Evolview v2: an online visualization and management tool for customized and annotated phylogenetic trees. Nucleic Acids Res. 2016;44(W1):W236-41. https://doi. org/10.1093/nar/gkw370.
37. Dai XB, Zhao PX. psRNATarget: a plant small RNA target analysis server. Nucleic Acids Res. 2011;39(Web Server issue):W155-9.

38. Smoot ME, Ono K, Ruscheinski J, Wang PL, Ideker T. Cytoscape 2.8: new features for data integration and network visualization. Bioinformatics. 2011 27(3):431-2. https://doi.org/10.1093/bioinformatics/btq675.

39. Livaka KJ, Schmittgenb TD. Analysis of relative gene expression data using real-time quantitative PCR and the 2 (-Delta Delta C (T)) method. Methods. 2001;25(4):402-8. https://doi.org/10.1006/meth.2001.1262.

\section{Publisher's Note}

Springer Nature remains neutral with regard to jurisdictional claims in published maps and institutional affiliations.
Ready to submit your research? Choose BMC and benefit from:

- fast, convenient online submission

- thorough peer review by experienced researchers in your field

- rapid publication on acceptance

- support for research data, including large and complex data types

- gold Open Access which fosters wider collaboration and increased citations

- maximum visibility for your research: over $100 \mathrm{M}$ website views per year

At BMC, research is always in progress.

Learn more biomedcentral.com/submissions 